\title{
Aberrant hypomethylation and overexpression of the eyes absent homologue 2 suppresses tumor cell growth of human lung adenocarcinoma cells
}

\author{
TANGXIN GAO ${ }^{1,2^{*}}$, SHANGYONG ZHENG ${ }^{1,2^{*}}$, QIAN LI $^{2}$, PENGZHAN RAN ${ }^{1}$, \\ LIJUAN SUN $^{1}$, YUNCANG YUAN ${ }^{2}$ and CHUNJIE XIAO ${ }^{1,2}$ \\ ${ }^{1}$ School of Medicine, Yunnan University; ${ }^{2}$ Key Laboratory of Molecular Genetics of Human Complicated Diseases, \\ Department of Education, Kunming, Yunnan 650091, P.R. China
}

Received March 30, 2015; Accepted June 15, 2015

DOI: $10.3892 /$ or.2015.4245

\begin{abstract}
The eyes absent homologue 2 (EYA2) is a dualfunctional transcription factor/phosphatase that plays a critical role in neoplasia. The precise effects of EYA2 remain elusive in non-small cell lung cancer (NSCLC). In the present study, we examined EYA2 expression in NSCLC cell lines and a normal pulmonary epithelial cell line. We found that EYA2 was aberrantly upregulated in the lung adenocarcinoma cells. Therefore, we studied the methylation status of the eya 2 gene in a lung adenocarcinoma cell line, a normal pulmonary epithelial cell line and lung adenocarcinoma tissues. Furthermore, the eya2 gene was knocked down in lung adenocarcinoma cells via RNA interference to investigate the regulatory role of EYA2; specifically, cell proliferation, cell cycle distribution, apoptosis, migration and invasive capacities were assessed in tje EYA2-knockdown cancer cells. The results showed that the aberrant hypomethylation and overexpression of the eya 2 gene were associated with lung adenocarcinoma oncogenesis. In addition, inhibition of EYA2 expression suppressed tumour cell growth by altering the proliferation, cell cycle distribution, apoptosis, migration and invasive capacities of the cells. These findings demonstrated that EYA2 functions as a stimulant
\end{abstract}

Correspondence to: Professor Chunjie Xiao, Key Laboratory of Molecular Genetics of Human Complicated Diseases, Department of Education, 2 Cuihu Road, Kunming, Yunnan 650091, P.R. China E-mail: chjxiao@ynu.edu.cn

${ }^{*}$ Contributed equally

Abbreviations: EYA2, eyes absent homologue 2 protein (Accession no. CAA71310); eya2, eyes absent homologue 2 gene (gene ID: 2139); NSCLC, non-small lung cancer; LAC, lung adenocarcinoma; SCLC, small cell lung cancer; CpGs, cytosine-guanine dinucleotides; TSS, transcriptional start site

Key words: eyes absent homologue 2 protein, non-small lung cancer, methylation, proliferation, apoptosis, migration, invasion in lung adenocarcinoma pathogenesis and may facilitate the development of novel diagnostic targets and therapy strategies for lung adenocarcinoma.

\section{Introduction}

Lung cancer is the most common cause of cancer-related mortality in humans and is characterised as either nonsmall cell lung cancer (NSCLC) or small cell lung cancer (SCLC) based on the histological type, size and appearance of the malignant cells (1). NSCLC cases comprise $\sim 80 \%$ of all lung cancer cases. NSCLC is a heterogeneous cancer that can be classified into three major subtypes: lung squamous carcinoma, lung adenocarcinoma (LAC) and large cell carcinoma (2). Common lung cancer treatment includes chemotherapy, surgery and radiotherapy. Due to the lack of an efficient diagnostic procedure for early-stage lung cancer the majority of lung cancer patients are diagnosed with end-stage disease, resulting in undesirable therapeutic outcomes. A biomarker-driven approach in the pre-metastatic phase could aid cancer diagnosis. However, current markers, such as Th19 fragment antigen 21-1 and neuron-specific enolase, have only aided in the diagnosis of $37.3 \%$ of lung cancer patients (3).

Recently, DNA methylation, a promising biomarker in cancer diagnosis, was found to be significantly associated with tumour formation. DNA methylation can regulate gene expression via complex mechanisms (4). For instance, hypermethylated cytosines inhibit the combination of DNA and trans-acting factors in the context of cytosine-guanine dinucleotides (CpGs), while demethylated cytosines promote this combination. In cancer cells, $\mathrm{CpG}$ islands located in the promoter of tumour-suppressor genes and 'housekeeping' genes become hypermethylated, which decreases the expression of these genes and in turn activates oncogenes (5-10). Lokk et al observed the hypermethylation of $496 \mathrm{CpGs}$ in 379 genes and hypomethylation of 373 CpGs in 335 genes in NSCLC (7). Aberrant DNA methylation reportedly serves as a predictive biomarker for the early-stage diagnosis of cancer (9-13).

The eyes absent (EYA) protein family is a component of a conserved regulatory network that is involved in cell-fate determination and is associated with cell proliferation and 
survival (14). They encode four EYA proteins in humans, including EYA2, which is required in the retinal determination network and is essential during development. The single nucleotide polymorphisms (SNPs) of the eya2 gene were found to be associated with the overall survival rate of NSCLC patients (15). Studies suggest that EYA2 is overexpressed in epithelial ovarian cancer, cervical carcinogenesis and breast cancers and its overexpression is correlated with poor prognosis, especially in breast cancer (16-18). A recent study revealed that the downregulation of EYA2 by tristetraprolin protein may reduce cell viability (19). The eya 2 gene is reportedly methylated in colorectal cancers but not in normal tissues (20), and epigenetic silencing of the eya2 gene has been shown to promote pancreatic tumour growth (21). As a dual-functional transcription factor/phosphatase, EYA2 has been found to regulate cell development, differentiation and mortality and play an important role in DNA damage and repair (22-24). However, the fragment of the eya2 gene that was used to conduct the methylation analysis in previous studies included only 200 bases of the 5'-UTR and little information is available on the methylation status of other CpGs in the eya2 gene. Thus, the precise effect of EYA2 in NSCLC remains unclear, and additional studies of the methylation levels of all $\mathrm{CpG}$ islands in the eya2 gene and the precise role of EYA2 in NSCLC are warranted.

In the present study, we evaluated the expression of EYA2 in NSCLC cell lines and a normal lung cell line to identify the association between EYA2 and oncogenesis in NSCLC. We then examined the methylation levels of the eya2 gene in a LAC cell line and human LAC tissues. The results suggest that the aberrant expression and methylation of the eya2 gene are associated with LAC oncogenesis. Transfecting LAC cells with small interfering RNA (siRNA) inhibited the expression of EYA2, and stable knockdown of this gene in cells was verified; these cells were utilised in further research. Furthermore, the proliferation, cell cycle, apoptosis, migration and invasion of cells were assessed. Our data indicated that the inhibition of EYA2 suppressed the growth of tumour cells by attenuating cell proliferation, arresting the cell cycle, increasing apoptosis and repressing cell migration and invasion. Our findings identified the eya 2 gene as a cancer-related gene and a potential diagnostic biomarker for LAC.

\section{Materials and methods}

Ethics statement. All cells used in the present study were purchased from the American Type Culture Collection and all lung cancer tissues used in the present study were obtained from the First Affiliated Hospital of Kunming Medical University. All experimental protocols were reviewed and approved by the Yunnan University Ethics Committee and all subjects involved in the present study provided informed consent.

Cell culture and sample. The human lung adenocarcinoma cell line A549 (CRM-CCL-185 ${ }^{\mathrm{TM}}$; ATCC, Manassas, VA, USA), the human large cell carcinoma cell line H661 (HTB-183D ${ }^{\mathrm{TM}}$; ATCC) and the human lung squamous carcinoma cell line SKMES1 (HTB-58 ${ }^{\mathrm{TM}}$; ATCC), were cultured in Dulbecco's modified Eagle's medium (DMEM) (SH30022; Thermo Fisher
Scientific, Waltham, MA, USA) supplemented with $10 \%$ foetal bovine serum (FBS, BI, AU, 04-12-1). The BEAS-2B cell line (CRL-9609 ${ }^{\mathrm{TM}}$; ATCC), a human normal pulmonary epithelial cell line, was cultured in RPMI-1640 (SH30027; Thermo Fisher Scientific) supplemented with $10 \%$ FBS, and 10 paraffinembedded lung adenocarcinoma tissues were obtained from the First Affiliated Hospital of Kunming Medical University. Participants in the present study group ranged from 42 to 61 years of age (mean age of males $n=4,52.25$ years; mean age of females $n=6,55.83$ years). The patients did not undergo any preoperative chemotherapy or radiotherapy.

Western blot analysis. The total protein of the A549 and BEAS-2B cells was isolated on ice with a Tissue or Cell Total Protein Extraction kit (BSP003; Sangon, Shanghai, China). For each sample, $80 \mu \mathrm{g}$ of total protein was electrophoresed on a $10 \%$ SDS-polyacrylamide gel followed by electrophoretic transfer to a polyvinylidene fluoride membrane. Immunoblotting was performed using an EYA2 (N-16)-specific goat polyclonal antibody (sc-15097; Santa Cruz Biotechnology, Inc., Santa Cruz, CA, USA). The results were visualised by chemiluminescence with Super Signal West Pico (34080; Thermo Fisher Scientific). The expression level of the target proteins was normalised to the expression of GAPDH (Affinity, USA). The expression data were obtained with ImageJ.

Quantitative real-time PCR. Total RNA was extracted from the cultured cells and reverse transcribed using the PrimeScript ${ }^{\mathrm{TM}}$ RT reagent kit with gDNA Eraser (RR047; Takara, Otsu, Shiga, Japan). The transcription levels of the eya2 gene were quantitatively analysed using real-time PCR (25) on a 7300 Real-Time PCR system with the SYBR Premix Ex Taq ${ }^{\mathrm{TM}}$ (RR420, Takara). The amount of target cDNA in each sample was measured by determining a fractional PCR threshold cycle number $(\mathrm{Ct})$ and estimated by interpolating from a standard curve. The standard curve was prepared from known amounts of the corresponding product with the same primer sets and was run on each PCR plate. The transcription levels of the target gene were normalised to the transcription of the GAPDH gene. The primer sequences are shown in Table II.

CpG island analysis. The CpG islands of the eya2 gene were analysed with the Methyl Primer Express v1.0 and MethPrimer software available online (http://www.urogene. org/methprimer/) as follows: a region of $>200$ bases with a $\mathrm{G}+\mathrm{C}$ content of at least $50 \%$ and a ratio of observed to statistically expected $\mathrm{CpG}$ frequencies of at least $0.6 \mathrm{CpG}$ dinucleotides (4).

DNA extraction and bisulphite modification. The DNA was extracted from the cells using the Wizard Genomic DNA purification kit (A1120; Promega, Madison, WI, USA), while the QIAamp DNA FFPE Tissue Kit (50) (56404; Qiagen, Hilden, Germany) was used to extract DNA from paraffin-embedded tissues $\left(2.4 \mathrm{~cm}^{3}\right.$ of tumour and matching paracancerous tissues). The DNA yield and purity were determined using a NanoDrop 2000 spectrophotometer. From each sample, $500 \mathrm{ng}$ of genomic DNA was bisulphite-modified using a Methyledge $^{\mathrm{TM}}$ bisulphite conversion system (1301; Promega) according to the manufacturer's recommendations. 
Table I. The CpG island of EYA2 PCR regions and primers.

\begin{tabular}{|c|c|c|c|}
\hline Fragment site & & Primer (5' to $\left.3^{\prime}\right)$ & Product size (bp) \\
\hline $\begin{array}{c}1 \\
(-163) \text { to }(-36)\end{array}$ & Stage-1 (2) & $\begin{array}{l}\text { Sense: GAATGTTAGTGTTATTATTGAGGTTTTT } \\
\text { Antisense: CCTCCCCACCCACCAAC }\end{array}$ & 128 \\
\hline $\begin{array}{c}2 \\
(-36) \text { to }(200)\end{array}$ & Stage-1 (2) & $\begin{array}{l}\text { Sense: GAGGTTGGGTTTTGGTTTTTA } \\
\text { Antisense: ACCCCTTCTCCTCCCTAAAC }\end{array}$ & 237 \\
\hline $\begin{array}{c}3 \\
(181) \text { to }(444)\end{array}$ & Stage-1 (2) & $\begin{array}{l}\text { Sense: GTTTAGGGAGGAGAAGGGGT } \\
\text { Antisense: AAAAAATCCCYATAAACAACTCC }\end{array}$ & 264 \\
\hline $\begin{array}{c}4 \\
(422) \text { to }(543)\end{array}$ & $\begin{array}{l}\text { Stage-1 } \\
\text { Stage-2 }\end{array}$ & $\begin{array}{l}\text { Sense: TTAGGGAGGAGAAGGGGT } \\
\text { Antisense: CCCTTATACCTTCCTAACCC } \\
\text { Sense: GGAGTTGTTTATYGGGATTTTT } \\
\text { Antisense: CCCTTATACCTTCCTAACCCT }\end{array}$ & 122 \\
\hline $\begin{array}{c}5 \\
(556) \text { to }(788)\end{array}$ & Stage-1 (2) & $\begin{array}{l}\text { Sense: TAGTAGAGTTTTTTTTTGGAAAGGT } \\
\text { Antisense: CCATAAACACTACCTAAAAACTTAAAT }\end{array}$ & 233 \\
\hline
\end{tabular}

Fragment 4 PCR stage-1 and stage 2 were performed with two different pairs of primers, otherwise the same pairs were used.

Table II. Reverse transcription and real-time quantitative PCR primers.

\begin{tabular}{lll}
\hline Gene & \multicolumn{1}{c}{ Sense primer (5' to 3') } & \multicolumn{1}{c}{ Antisense primer (5' to 3') } \\
\hline EYA2 & CAAGGAGGAAATGGACTGGG & GGGTTGTAGGATGAGCCGTAA \\
GAPDH & CACTCCTCCACCTTTGACGC & TGCTGTAGCCAAATTCGTTGT \\
\hline
\end{tabular}

Reverse transcription was performed using antisense primers and real-time quantitative PCRs were performed using both sense primers and antisense primers.

Bisulphite genomic-PCR. The $\mathrm{CpG}$ islands of the eya2 gene were produced using nested PCR (26) and detected with agarose gel electrophoresis. The modified DNA was subjected to stage-1 PCR with Tm touchdown from 68 to $48^{\circ} \mathrm{C}$, followed by 20 cycles with $\mathrm{Tm}$ of $48^{\circ} \mathrm{C}$. The product of the stage was then used as a template for the stage 2 PCR. Six pairs of primers (Table I; fragment 1, 2, 3, 4 and 5) were used for the $\mathrm{CpG}$ island of the eya2 gene in the cell lines and four pairs of primers (Table I; fragment 1,2,3 and 5) were used for the tissues. The product DNA was visualised on $2 \%$ agarose gels and purified with the Takara MiniBEST Agarose Gel DNA Extraction kit ver. 4.0 (9762; Takara).

DNA cloning and sequencing. The purified CpG island DNA was cloned with the pMDTM18-T vector cloning kit (Takara, 6011) and Dh5 $\alpha$ competent cells (Takara, 9057). The clone products were sequenced at the Beijing Genomics Institute (BGI, Shenzhen, China). The gene methylation status was analysed with the BIQ Analyser (27).

Liposome-mediated lung adenocarcinoma cell transfection. The experiment was divided into three groups: the EYA2-special siRNA-FAM A549 cell group, the negative control-FAM A549 cell group (scrambled) and the untreated
A549 cell group (mock). EYA2-specific siRNA sequences were forward, CAGCGAUUGUCUGGAUAAATT and reverse, UUUAUCCAGACAAUCGCUGTT. The scrambled siRNA sequences were forward, UUCUCCGAACGUGUC ACGUTT and reverse, ACGUGACACGUUCGGAGAATT (A10005; GenePharma, Shanghai, China). For liposome transfection, the cells were plated in 6-well plates (1x10/well) $12 \mathrm{~h}$ prior to transfection. All siRNAs (5 $\mu \mathrm{g} /$ well) were transfected with Lipofectamine 3000 (L3000001; Invitrogen, Carlsbad, CA USA).

Cell proliferation assay. The cell viability was evaluated with a 3-(4,5-dimethylthiazol-2-yl)-5-(3-carboxymethoxyphenyl)2-(4-sulfophenyl)-2H-tetrazolium inner salt (MTS) colorimetric assay using the CellTiter 96 Aqueous One Solution Cell Proliferation Assay (G3582; Promega) according to the manufacturer's instructions. All groups were dispensed at a density of 1,000 cells/well in 96-well plates with DMEM containing 10\% FBS and the corresponding transfection solution after transfection for $48 \mathrm{~h}$. After various intervals of incubation, $40 \mu 1$ of MTS reagent was added to the medium and incubated for $4 \mathrm{~h}$ at $37^{\circ} \mathrm{C}$ in $5 \% \mathrm{CO}_{2}$ and the absorbance was then read at $490 \mathrm{~nm}$ in a microplate absorbance reader. 
Cell cycle and apoptosis analysis. To analyse the cell cycle, the cells were collected $48 \mathrm{~h}$ after transfection. The cells were then washed with PBS and stained with $50 \mu \mathrm{g} / \mathrm{ml}$ propidium iodide (PI) in the dark at room temperature for $5 \mathrm{~min}$. The intracellular DNA content was analysed using a flow cytometry (Accuri $^{\mathrm{TM}} \mathrm{C} 6$; BD).

To analyse apoptosis, the apoptotic cells were quantified using flow cytometry $72 \mathrm{~h}$ after transfection using an Annexin V-FITC/PI Apoptosis Detection kit (556547; BD) according to the manufacturer's instructions. The cells were collected, washed with PBS and re-suspended with $100 \mu \mathrm{l}$ of $1 \mathrm{X}$ Annexin V-binding buffer. The samples were then stained with $5 \mu \mathrm{l}$ of FITC Annexin V and $5 \mu \mathrm{l}$ of PI at room temperature for $15 \mathrm{~min}$ in the dark. In addition, $400 \mu \mathrm{l} 1 \mathrm{X}$ Annexin V-binding buffer was added prior to the flow cytometric analysis.

Migration assay. The migratory ability of the A549 cells was evaluated using the wound healing method, as previously described (28). Three groups of cells (transfected with 0,100 or $200 \mathrm{nM}$ siRNA) were grown to confluency in 6-well plates. A line of cells was scraped away in each well using a sterile $200 \mu 1$ pipette tip. The wells were then immediately filled with $1 \mathrm{ml}$ of DMEM containing 10\% FBS and the corresponding transfection reagent.

Immediately after the scratch and at $24 \mathrm{~h}$, two images of five locations of the same scraped area were captured with a microscope set at a magnification $\times 100$. The remaining wounded area per image was measured. Three independent experiments were performed.

Invasion assay. The invasive potential of the cells was assessed in a Transwell, which allowed the cells to pass through a polycarbonate membrane $(8-\mu \mathrm{m}$ pore size) coated with Matrigel (356234; Haoran, Shanghai, China). Briefly, the Transwell was coated with Matrigel $(2 \mathrm{mg} / \mathrm{ml})$ diluted with serum-free DMEM for $30 \mathrm{~min}$ at $37^{\circ} \mathrm{C}$. Three groups of cells (transfected with 0,100 or $200 \mathrm{nM}$ siRNA) $\left(5 \times 10^{4}\right.$ cells) were then re-suspended in serum-free DMEM $(200 \mu \mathrm{l})$ and deposited into the upper chamber of each well. The lower chambers were also filled with $750 \mu 1$ of DMEM containing 10\% FBS. The cells were incubated for $24 \mathrm{~h}$ at $37^{\circ} \mathrm{C}$ in $5 \% \mathrm{CO}_{2}$. The cells on the upper chamber were removed via gentle scraping. The cells on the lower surface were then fixed with $4 \%$ paraformaldehyde for $20 \mathrm{~min}$, followed by Giemsa staining for $20 \mathrm{~min}$ in the dark. Four images of the invading cells were captured at a magnification $\times 100$ in each well. Three independent experiments were performed.

Statistical analysis. Statistical analysis was carried out using the GraphPad Prism software (version 5.0) (29), and all results are expressed as the mean $\pm \mathrm{SD}$. A t-test was performed to identify the differences in the DNA methylation levels of the eya 2 gene between the A549 and BEAS-2B cells. A one-way ANOVA was performed to identify the differences in expression intensities of the EYA2 protein among four groups of examined cells, changes in proliferation, the cell cycle, apoptosis, migration capacity and invasive capability after the knockdown of EYA2 in A549 cells. P-values of $<0.05$ were considered statistically significant.
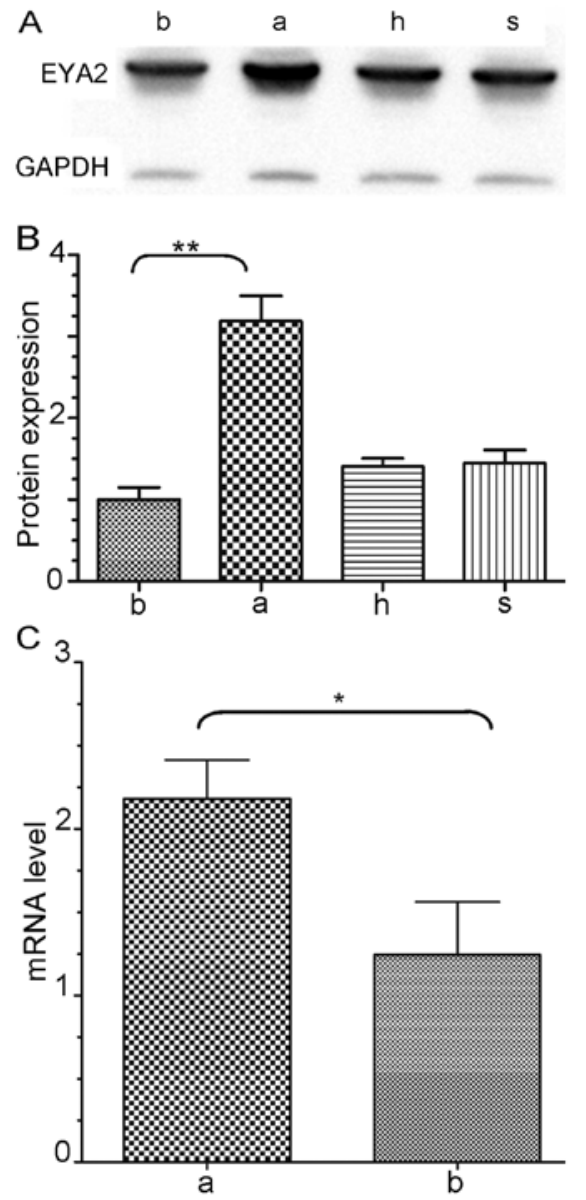

Figure 1. Expression of EYA2 in NSCLC cells. (A) Western blot analysis (B) EYA2 protein expression was significantly upregulated 3.2-fold in the A549 cells compared to the BEAS-2B cells, but had no obvious change in the H661 and SKMES1 cells. (C) mRNA level of EYA2 exhibited a 2.1-fold upregulation in the A549 cells compared to the BEAS-2B cells. The groups were obtained from different parts of the same gel. ${ }^{*} \mathrm{P}<0.05$ and ${ }^{* *} \mathrm{P}<0.01$. a A549; b, BEAS-2B; h, H661; s, SKMES1 cells.

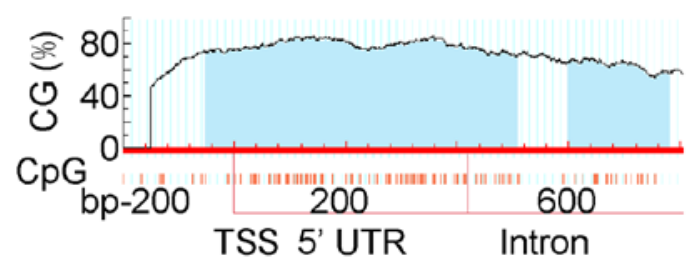

Figure 2. The CpG Islands of EYA2. The exhibited sequence was from the transcription initiation site (TSS) $200 \mathrm{bp}$ upstream to $958 \mathrm{bp}$ downstream. The CpG islands and $\mathrm{CpG}$ dinucleotide density are each depicted in this figure: $\mathrm{CpG}$ islands (blue regions), $\mathrm{CpG}$ dinucleotides (red vertical bar).

\section{Results}

Upregulated expression of EYA2 in lung adenocarcinoma cells. The EYA2 protein expression levels were quantified (Fig. 1A). The results of the western blot analysis revealed that the expression of EYA2 protein was significantly upregulated 3.2-fold in the A549 cells compared to that in the BEAS-2B cells $(\mathrm{P}<0.01$, Fig. 1B), while obvious differences were absent in the H661 and SKMES1 cells compared to that in the BEAS-2B cells. The mRNA and protein levels were detected 
A

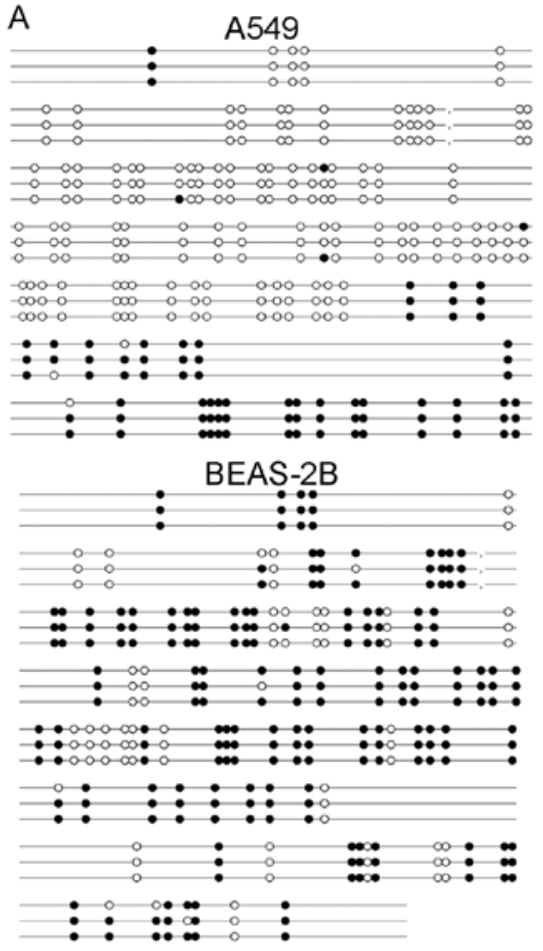

B

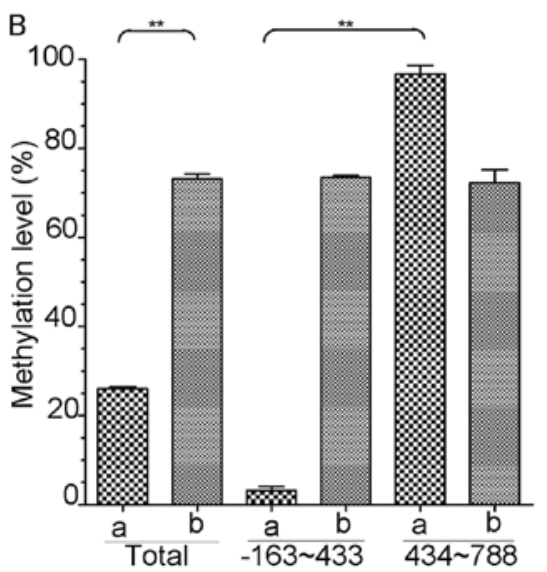

D

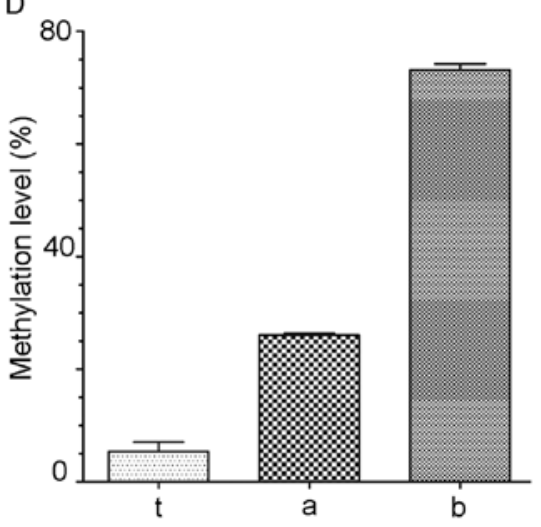

C
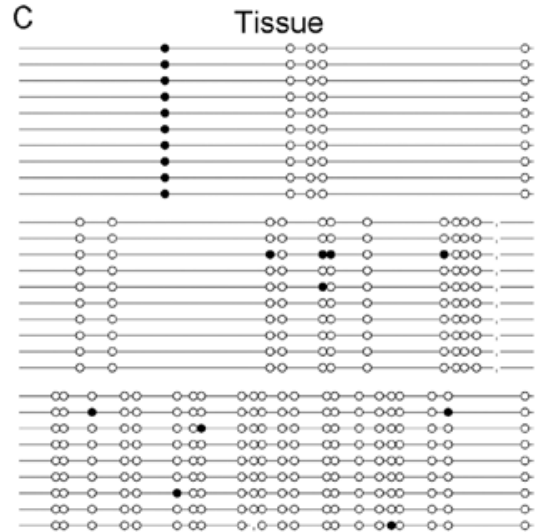

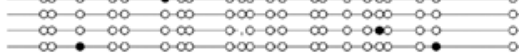
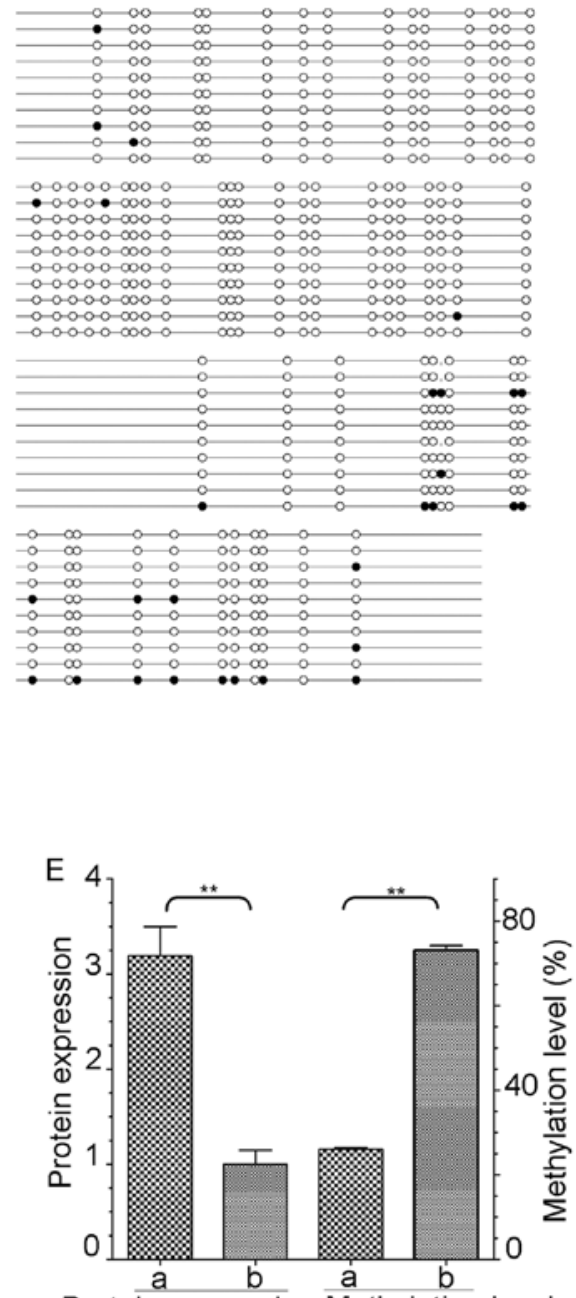

Protein expression Methylation level

Figure 3. Aberrant methylation status in LAC. Filled (black) circles correspond to methylated Cs, unfilled (white) circles correspond to unmethylated Cs and small vertical lines without a circle correspond to missing values. ${ }^{* *} \mathrm{P}<0.01$. a, A549; b, BEAS-2B; t, LAC tissues.

by quantitative real-time RT-PCR and western blot analysis, respectively ( $\mathrm{n}=3 /$ group). The EYA2 mRNA transcriptional level was upregulated 2.1-fold in the A549 cells compared to this level in the BEAS-2B cells and this upregulation was significant $(\mathrm{P}<0.05$, Fig. $1 \mathrm{C})$.
Aberrant hypomethylation of the eya 2 gene in lung adenocarcinoma. The $\mathrm{CpG}$ islands of the eya 2 gene are shown in Fig. 2. The entire eya 2 gene sequence, including the 2000-bp upstream region of the TSS, was analysed. CpG islands were not found upstream of TSS and a long fragment of $951 \mathrm{bp}$ 

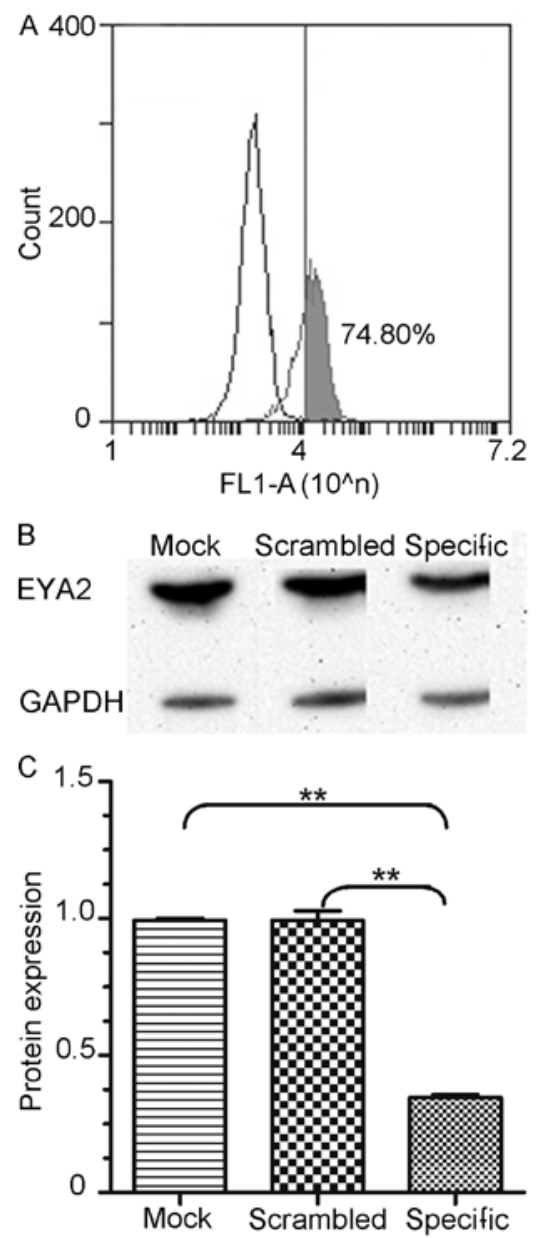

Figure 4. EYA2 was knocked down by siRNA in A549 cells. (A) The gray area represents successfully transfected cells and transfection efficiency was detected by flow cytometry. Cells $(74.80 \%)$ were successfully transfected with siRNA-FAM. (B) The knockdown efficiency of EYA2 was examined by western blot analysis. (C) The differences between the specific and mock construct and between the specific and scrambled construct were both significant ( 0.3472 vs. 0.9934 and 0.3472 vs. 0.9925$)$. The groups were obtained on different parts of the same gel. ${ }^{* *} \mathrm{P}<0.01$.

(from the $163 \mathrm{bp}$ upstream to the $788 \mathrm{bp}$ downstream of the eya 2 gene TSS, containing the upstream region of TSS, 5'-UTR and the first intron) that included $105 \mathrm{CpGs}$ was selected. The selected area was divided into 5 DNA fragments to perform PCR. Five fragment locations and the primers for PCR are listed in Table I.

A total of three clones were detected in the two cell lines. The methylation statuses of the eya2 gene in the A549 and BEAS-2B cells are described (Fig. 3A). The data were corrected for reversed sequences, erroneous bases and identical clones. On average $27 \mathrm{CpG}$ dinucleotides out of $105 \mathrm{CpGs}$ were methylated in the A549 cells and $76 \mathrm{CpGs}$ out of $105 \mathrm{CpGs}$ were methylated in the BEAS-2B cells. The total methylation level of $\mathrm{CpGs}$ significantly differed between the A549 and BEAS-2B cells at 25.71 and $72.38 \%$, respectively ( $\mathrm{P}<0.01$, Fig. 3B). Moreover, the methylation level of the A549 cells significantly varied by fragment. Two out of $75 \mathrm{CpGs}$ were methylated in the TSS 163-bp upstream to 433-bp downstream fragment and 30 CpGs were methylated in the TSS 434 to 788 bp downstream fragment; the overall methylation levels were 2.67 and $96.67 \%$, respectively $(\mathrm{P}<0.01)$. These two frag- ments did not significantly differ and the methylation levels were 73.43 and $72.23 \%$ in the BEAS-2B cells.

In total, 10 tumour tissues were detected by BSP. The methylation statuses of the tissues are displayed in Fig. 3C, and the data were corrected for reversed sequences, erroneous bases and identical clones. On average, $5 \mathrm{CpG}$ dinucleotides out of $96 \mathrm{CpGs}$ were methylated in the tumour tissues (4 fragments, including $9 \mathrm{CpGs}$, were excluded because most of the tumour tissues did not yield PCR products). The total methylation levels of the eya2 gene were low in both the A549 cells and tumour tissues compared with the level in the BEAS-2B cells (Fig. 3D). However, the methylation levels in the tumour tissues were lower than those in the A549 cells at 5.31 and $25.71 \%$, respectively. The obvious difference between the TSS 163-bp upstream to 433-bp downstream fragment and the TTS 434 to 788 bp downstream fragment was absent in the tumour tissues.

To research the significance of EYA2 in LAC, the relevance of EYA2 expression to the methylation status of the eya 2 gene was analysed in the A549 and BEAS-2B cells. The distribution of the eya2 gene methylation levels was inversely correlated with the EYA2 expression levels in these two cell lines (Fig. 3E).

Knockdown of EYA2 effects lung adenocarcinoma cell proliferation by arresting the cell cycle and increasing apoptosis. To further investigate the biological role of EYA2 in LAC, an RNA interference approach was utilised to downregulate the expression of EYA2 in the A549 cell line. Moreover, we evaluated the knockdown efficiencies of EYA2-specific siRNA-FAM in the A549 cells by flow cytometry and western blot analysis ( $\mathrm{n}=3$ /group) $48 \mathrm{~h}$ after transfection. We detected that $74.80 \%$ of the processed cells were successfully transfected with siRNA-FAM (Fig. 4A). The expression level of EYA2 was significantly decreased in the cells transfected with the EYA2-specific siRNA $48 \mathrm{~h}$ after transfection compared with the negative control and the untreated group (Fig. 4B). The differences between the specific and mock construct and between the specific and scrambled construct were both significant $(0.3472$ vs. $0.9934, \mathrm{P}<0.01$ and 0.3472 vs. $0.9925, \mathrm{P}<0.01$; Fig. 4C). These results indicated that the EYA2-specific siRNA effectively suppressed EYA2 expression.

We assessed the effects of EYA2 knockdown on the proliferation of A549 cells with an MTS colorimetric assay ( $n=5 /$ group). Our data revealed that the inhibition of EYA2 significantly inhibited the proliferation of the A549 cells (Fig. 5A). Our findings demonstrate that EYA2 contributed to the proliferation of A549 cells in vitro.

Because the knockdown of EYA2 by siRNA suppressed the proliferation of A549 cells, we examined whether this effect was mediated by a change in the cell cycle. We detected changes in the cell cycles in the three groups of siRNA-treated cells (specific, scrambled and mock) $48 \mathrm{~h}$ after transfection using PI staining and flow cytometry ( $n=3 /$ group) (Fig. 5B). The number of cells in the G0/G1 phase was significantly increased and those in the $\mathrm{S}$ and $\mathrm{G} 2 / \mathrm{M}$ phases were significantly decreased in the cells transfected with the specific siRNA compared to the mock group and the scrambled group (G0/ G1: 85.56 vs. $77.95 \%, \mathrm{P}<0.01$ and 85.56 vs. $78.25 \%, \mathrm{P}<0.05$; $\mathrm{S}$ : 11.25 vs. $16.65 \%, \mathrm{P}<0.05$ and 11.25 vs. $16.25 \%, \mathrm{P}<0.05 ; \mathrm{G} 2 / \mathrm{M}$ : 

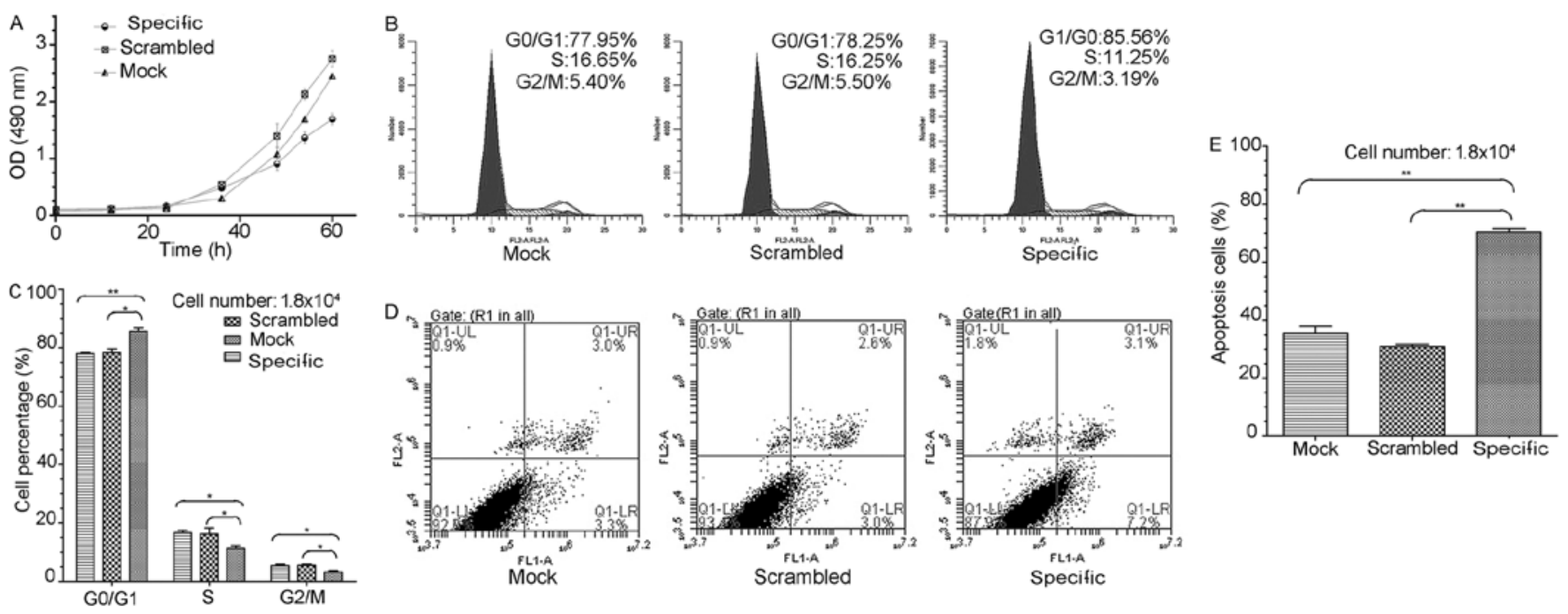

Figure 5. Knockdown of EYA2 inhibits the growth of the A549 cells. (A) The cell proliferation was determined with an MTT assay and is presented as the absorbance values at $490 \mathrm{~nm}$. EYA2 silencing suppressed the proliferation of the A549 cells. (B) Changes in the cell cycle were detected $48 \mathrm{~h}$ after transfection using PI staining and flow cytometry. (C) The percentage of cells in the G0/G1 phase was significantly increased and the percentages in the S and G2/M phases were significantly decreased in the cells transfected with specific siRNA compared to the mock and the scrambled group (G0/G1, 85.56 vs. 77.95 and 85.56 vs. $78.25 ; \mathrm{S}, 11.25$ vs. 16.65 and 11.25 vs. $16.25 ; \mathrm{G} 2 / \mathrm{M}, 3.19$ vs. 5.40 and 3.19 vs. 5.5\%). (D) The differences in cell apoptosis were tested 60-72 h after transfection using Annexin V-FITC and PI double staining as well as flow cytometry. (E) The percentage of apoptotic cells in the specific group was markedly increased compared to the percentages in the scrambled group and the mock group ( 7.2 vs. 3.0 and 7.2 vs. $3.3 \%$ ). ${ }^{*} \mathrm{P}<0.05$ and ${ }^{* * *} \mathrm{P}<0.01$.
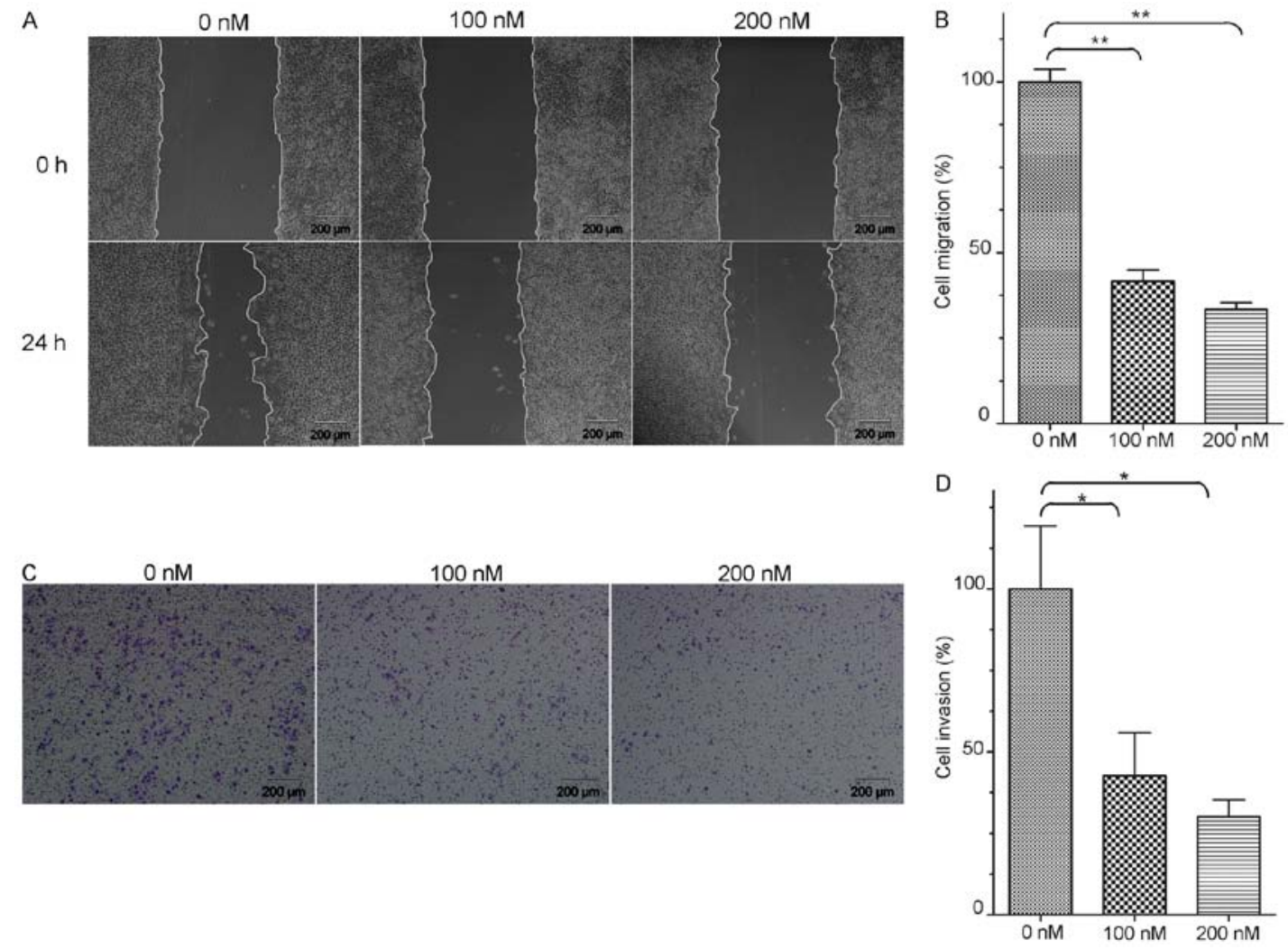

Figure 6. Knockdown of EYA2 suppresses the migration and invasion of the A549 cells. (A) A wound healing assay was used to detect changes in the migration capabilities of the A549 cells. (B) The percentage (vs. control) of A549 cells that penetrated the membrane was significantly decreased by 41.70 and $33.41 \%$ compared with the control group in response to transfection with 100 and $200 \mathrm{nM}$ siRNA, respectively. (C) The invasive capacity of the A549 cells after the knockdown of EYA2 was tested. (D) After transfection with 100 and $200 \mathrm{nM}$ siRNA for $24 \mathrm{~h}$, the abilities of the cells to enzymatically degrade the Matrigel and migrate to a site was significantly decreased at an invasive percentage (vs. control) of 42.63 and $30.13 \%$ compared with the control group, respectively. ${ }^{*} \mathrm{P}<0.05$ and ${ }^{* *} \mathrm{P}<0.01$. 
To explore the potential effect of the knockdown of EYA2 on apoptosis in the A549 cells, we examined differences in apoptosis between the three siRNA-transfected groups of cells (specific, scrambled and mock) 60-72 $\mathrm{h}$ after transfection using Annexin V-FITC and PI double-staining in conjunction with flow cytometry (n=3/group) (Fig. 5D). The percentage of apoptotic cells in the specific group was markedly increased compared to the scrambled group and the mock group (7.2 vs. $3.0 \%, \mathrm{P}<0.01$ and 7.2 vs. $3.3 \%, \mathrm{P}<0.01$; Fig. $5 \mathrm{E}$ ). These results imply that the inhibition of EYA2 promoted the apoptosis of the A549 cells.

Downregulation of EYA2 inhibits the migratory and invasive potential of lung adenocarcinoma cells. A wound healing assay (WH) was used to detect the change in the migratory capability of the A549 cells (Fig. 6A). After transfection with 100 and $200 \mathrm{nM}$ siRNA for $24 \mathrm{~h}$, the percentages of A549 cells that penetrated the membrane were significantly decreased by 41.70 and $33.41 \%$ compared with that in the control group, respectively ( $\mathrm{P}<0.01$, Fig. $6 \mathrm{~B})$. These results indicated that the downregulation of EYA2 suppressed the migratory capacity of the A549 cells.

Additionally, we tested the invasive capacity of the A549 cells after the knockdown of EYA2 (Fig. 6C). After transfection with 100 and $200 \mathrm{nM}$ siRNA for $24 \mathrm{~h}$, the abilities of the cells to enzymatically degrade the Matrigel and migrate to a new site was significantly decreased by 42.63 and $30.13 \%$ compared with the control group ( $\mathrm{P}<0.05$, Fig. 6D). Therefore, the results revealed that the knockdown of EYA2 decreased the invasive capacity of the A549 cells.

\section{Discussion}

EYA2 is a key regulator that can prevent adverse cardiac remodelling under pressure overload by altering metabolic gene expression and preserving the $\mathrm{PI} 3 \mathrm{~K} / \mathrm{Akt} / \mathrm{mTOR}$ signalling pathway $(30,31)$. Furthermore, the physical complex of EYA2 and SIX1 plays a critical role in the preservation of mitochondrial integrity in response to pressure overload due to physiological hypertrophy and is essential for mammalian development (32); a loss of this function may cause branchiootorenal (BOR) syndrome $(33,34)$. Moreover, EYA2 is associated with $\mathrm{G}$ protein-mediated signalling, retinoid-induced limb malformations and hypaxial somitic myogenesis (35-37). Thus, EYA2 may exert a key effect on disease development and tumourigenesis. In the present study, we analysed the expression of EYA2 in NSCLC cells and studied the methylation of the eya2 gene in the A549 and BEAS-2B cell lines as well as in LAC tissues. In addition, we knocked down the expression of EYA2 and conducted various functional studies associated with tumour cell growth and metastasis in these cells.

Farabaugh et al found that EYA2 functions as a prometastatic factor in breast cancer (17). Vincent and his collaborators hypothesised that EYA2 expression is reduced due to epigenetic silencing, which promotes tumour growth in pancreatic cancer (21). Zou et al reported that EYA2 is hypermethylated in colorectal neoplasms (20). In the present study, the eya2 gene was hypomethylated in the A549 cell line and cancer tissues and hypermethylated in the BEAS-2B cell line. The results showed that the DNA methylation levels significantly differed between the LAC and the normal control samples. Moreover, the selected intron region significantly differed between the paraffin-embedded tissues and the A549 cell line. The growth environment of the A549 cell line in vitro differs from the in vivo environment and further research is warranted to determine whether the developmental conditions of the cells influence their DNA methylation levels. Furthermore, the EYA2 expression was markedly increased in the lung adenocarcinoma cell line compared with the normal pulmonary epithelial cell line and the relevance of the methylation levels of the eya2 gene and EYA2 expression levels were markedly inversely correlated, which is consistent with a study by Zhang et al (18). In contrast to previous studies in which the methylation levels of short sequences of the eya 2 gene 5'-UTR region were analysed, we analysed the methylation status of the entire $\mathrm{CpG}$ islands of the eya 2 gene, which containing almost all selected fragments in previously methylated studies of the eya 2 gene. Thus, our data constitute conclusive proof of the association between the aberrant methylation of EYA2 and oncogenesis in NSLCL. The function of EYA2 may be organ- or cell-specific. Further studies are warranted to elucidate the complex function of EYA2 in tumours.

Additionally, deregulated cell cycle progression is one of the primary characteristics of human cancer cells (38). EYA2 was found to increase the expression of c-Myc, cyclin A, D1 and $\mathrm{E}$, which are all involved in $\mathrm{G} 1 / \mathrm{S}$ cell cycle progression in breast cancer cells (39). Clark et al discovered that inappropriate changes in the steady state levels of EYA proteins can trigger programmed cell death during development (40). To identify the role that EYA2 plays in the function of A549 cells, we knocked down the expression of EYA2 in A549 cells via RNA interference technology in vitro. First, we confirmed that the specific siRNA could efficiently reduce the EYA2 expression in the A549 cells. The proliferation rate of the A549 cells decreased when the endogenous EYA2 was downregulated compared with the control groups. Moreover, we examined the cycle distribution of the A549 cells after the inhibition of EYA2. Our data revealed that the knockdown of EYA2 arrested cells in the G0/G1 phase. Furthermore, the percentage of apoptotic A549 cells was increased when EYA2 was knocked down compared with the control group. Thus, we concluded that reduction of EYA2 may suppress A549 cell proliferation by inhibiting the G1/S transition of the cell cycle and increasing apoptosis. In addition, previous studies also showed that EYA2 is associated with cell proliferation, the cell cycle and apoptosis (41-44).

Pandey et al discovered that EYA3, which belongs to the EYA family of proteins, promoted not only tumour cell proliferation, but also their migration and invasion (45). EYA2 is a homologous protein of EYA3 and may play the same important role in tumour cell metastasis. Therefore, we investigated the effect of EYA2 knockdown on cell migration and invasion in the A549 cells. As expected, downregulation of EYA2 reduced the migratory and invasive capacities of the A549 cells, consistent with prior research by Krueger et al, who implicated EYA2 in tumour development (46).

In conclusion, we found that the methylation status and expression of the eya 2 gene were altered in lung 
adenocarcinoma and that the knockdown of EYA2 could change various functions in lung adenocarcinoma cells. Previous studies have shown that the eya2 gene may serve as a promising early-stage diagnostic biomarker and key treatment target gene in lung adenocarcinoma. These findings imply the importance of EYA2 in NSCLC development and progression. We plan to explore whether the growth status of tumour cells can deteriorate by specifically modulating the methylation of the eya 2 gene. To this end, a large number of specific inhibitors should be screened to ameliorate the aberrant expression of EYA2 in tumour cells and identify an inhibitor that can suppress the growth of tumour cells. Thus, clearly further investigation are required.

\section{Acknowledgements}

We express our sincere appreciation to all patients that participated in the present study. The present study was financially supported by grants from the National Natural Science Foundation of China (no. 81372360) to C.X. and from the National Natural Science Foundation of China (no. 81460435) and the Key Projects of Applied Basic Reasearch of Yunnan Province (no. 2014FA022) to S.Z.

\section{References}

1. Kumar V: Abass KA, Fausto N and Mitchell R: Robbins Basic Pathology. 8th edition, Saunders Elsevier, Philadelphia, PA, 2007.

2. Brambilla E, Travis WD, Colby TV, Corrin B and Shimosato Y: The new World Health Organization classification of lung tumours. Eur Respir J 18: 1059-1068, 2001.

3. Chu XY, Hou XB, Song WA, Xue ZQ, Wang B and Zhang LB: Diagnostic values of SCC, CEA, Cyfra21-1 and NSE for lung cancer in patients with suspicious pulmonary masses: A single center analysis. Cancer Biol Ther 11: 995-1000, 2011.

4. Portela A and Esteller M: Epigenetic modifications and human disease. Nat Biotechnol 28: 1057-1068, 2010.

5. Takai D and Jones PA: Comprehensive analysis of CpG islands in human chromosomes 21 and 22. Proc Natl Acad Sci USA 99: 3740-3745, 2002.

6. Grønbaek K, Hother $\mathrm{C}$ and Jones PA: Epigenetic changes in cancer. APMIS 115: 1039-1059, 2007.

7. Lokk K, Vooder T, Kolde R, Välk K, Võsa U, Roosipuu R, Milani L, Fischer K, Koltsina M, Urgard E, et al: Methylation markers of early-stage non-small cell lung cancer. PLoS One 7: e39813, 2012.

8. Tekpli X, Landvik NE, Anmarkud KH, Skaug V, Haugen A and Zienolddiny $\mathrm{S}$ : DNA methylation at promoter regions of interleukin $1 \mathrm{~B}$, interleukin 6 , and interleukin 8 in non-small cell lung cancer. Cancer Immunol Immunother 62: 337-345, 2013.

9. Sato T, Arai E, Kohno T, Tsuta K, Watanabe S, Soejima K, Betsuyaku T and Kanai Y: DNA methylation profiles at precancerous stages associated with recurrence of lung adenocarcinoma. PLoS One 8: e59444, 2013.

10. Pesta M, Kulda V, Topolcan O, Safranek J, Vrzalova J, Cerny R and Holubec L: Significance of methylation status and the expression of RECK mRNA in lung tissue of patients with NSCLC. Anticancer Res 29: 4535-4539, 2009.

11. Suzuki M, Shiraishi K, Eguchi A, Ikeda K, Mori T, Yoshimoto K, Ohba Y, Yamada T, Ito T, Baba Y, et al: Aberrant methylation of LINE-1, SLIT2, MAL and IGFBP7 in non-small cell lung cancer. Oncol Rep 29: 1308-1314, 2013.

12. Grimminger PP, Maus MK, Schneider PM, Metzger R, Hölscher AH, Sugita H, Danenberg PV, Alakus H and Brabender J: Glutathione S-transferase PI (GST-PI) mRNA expression and DNA methylation is involved in the pathogenesis and prognosis of NSCLC. Lung Cancer 78: 87-91, 2012.
13. Duan H, He Z, Ma J, Zhang B, Sheng Z, Bin P, Cheng J, Niu Y, Dong H, Lin H, et al: Global and MGMT promoter hypomethylation independently associated with genomic instability of lymphocytes in subjects exposed to high-dose polycyclic aromatic hydrocarbon. Arch Toxicol 87: 2013-2022, 2013.

14. Tadjuidje E and Hegde RS: The eyes absent proteins in development and disease. Cell Mol Life Sci 70: 1897-1913, 2013.

15. Huang YT, Heist RS, Chirieac LR, Lin X, Skaug V, Zienolddiny S, Haugen A, Wu MC, Wang Z, Su L, et al: Genome-wide analysis of survival in early-stage non-small-cell lung cancer. J Clin Oncol 27: 2660-2667, 2009.

16. Bierkens M, Krijgsman O, Wilting SM, Bosch L, Jaspers A, Meijer GA, Meijer CJ, Snijders PJ, Ylstra B and Steenbergen RD: Focal aberrations indicate EYA2 and hsa-miR-375 as oncogene and tumor suppressor in cervical carcinogenesis. Genes Chromosomes Cancer 52: 56-68, 2013.

17. Farabaugh SM, Micalizzi DS, Jedlicka P, Zhao R and Ford HL: Eya 2 is required to mediate the pro-metastatic functions of Six 1 via the induction of TGF- $\beta$ signaling, epithelial-mesenchymal transition, and cancer stem cell properties. Oncogene 31: 552-562, 2012.

18. Zhang L, Yang N, Huang J, Buckanovich RJ, Liang S, Barchetti A, Vezzani C, O'Brien-Jenkins A, Wang J, Ward MR, et al: Transcriptional coactivator Drosophila eyes absent homologue 2 is up-regulated in epithelial ovarian cancer and promotes tumor growth. Cancer Res 65: 925-932, 2005.

19. Yeh PA, Yang WH, Chiang PY, Wang SC, Chang MS and Chang CJ: Drosophila eyes absent is a novel mRNA target of the tristetraprolin (TTP) protein DTIS11. Int J Biol Sci 8: 606-619, 2012.

20. Zou H, Harrington JJ, Shire AM, Rego RL, Wang L, Campbell ME, Oberg AL, Ahlquist DA: Highly methylated genes in colorectal neoplasia: implications for screening. Cancer Epidemiol, Biomarkers Prev 16: 2686-2696, 2007.

21. Vincent A, Hong SM, Hu C, Omura N, Young A, Kim H, Yu J, Knight S, Ayars M, Griffith M, et al: Epigenetic silencing of EYA2 in pancreatic adenocarcinomas promotes tumor growth. Oncotarget 5: 2575-2587, 2014.

22. Jemc J and Rebay I: Identification of transcriptional targets of the dual-function transcription factor/phosphatase eyes absent. Dev Biol 310: 416-429, 2007.

23. Jemc $\mathrm{J}$ and Rebay I: The eyes absent family of phosphotyrosine phosphatases: Properties and roles in developmental regulation of transcription. Annu Rev Biochem 76: 513-538, 2007.

24. Krishnan N, Jeong DG, Jung SK, Ryu SE, Xiao A, Allis CD, Kim SJ and Tonks NK: Dephosphorylation of the C-terminal tyrosyl residue of the DNA damage-related histone $\mathrm{H} 2 \mathrm{~A}$.X is mediated by the protein phosphatase eyes absent. J Biol Chem 284: 16066-16070, 2009.

25. Spackman E and Suarez DL: Type A influenza virus detection and quantitation by real-time RT-PCR. Methods Mol Biol 436: 19-26, 2008.

26. Kobayashi H and Kono T: DNA methylation analysis of germ cells by using bisulfite-based sequencing methods. Methods Mol Biol 825: 223-235, 2012.

27. Becker D, Lutsik P, Ebert P, Bock C, Lengauer T and Walter J: BiQ Analyzer HiMod: An interactive software tool for high-throughput locus-specific analysis of 5-methylcytosine and its oxidized derivatives. Nucleic Acids Res 42 (W1): W501-W507, 2014.

28. Arranz-Valsero I, Soriano-Romaní L, García-Posadas L, López-García A and Diebold Y: IL-6 as a corneal wound healing mediator in an in vitro scratch assay. Exp Eye Res 125: 183-192, 2014.

29. Guo J, Lin P, Zhao X, Zhang J, Wei X, Wang Q and Wang C: Etazolate abrogates the lipopolysaccharide (LPS)-induced downregulation of the $\mathrm{cAMP} / \mathrm{pCREB} / \mathrm{BDNF}$ signaling, neuroinflammatory response and depressive-like behavior in mice. Neuroscience 263: 1-14, 2014.

30. Yang DK, Choi BY, Lee YH, Kim YG, Cho MC, Hong SE, Kim H, Hajjar RJ and Park WJ: Gene profiling during regression of pressure overload-induced cardiac hypertrophy. Physiol Genomics 30: 1-7, 2007.

31. Lee SH, Yang DK, Choi BY, Lee YH, Kim SY, Jeong D, Hajjar RJ and Park WJ: The transcription factor Eya2 prevents pressure overload-induced adverse cardiac remodeling. J Mol Cell Cardiol 46: 596-605, 2009.

32. Lee SH, Kim J, Ryu JY, Lee S, Yang DK, Jeong D, Kim J, Lee SH, Kim JM, Hajjar RJ, et al: Transcription coactivator Eya2 is a critical regulator of physiological hypertrophy. J Mol Cell Cardiol 52: 718-726, 2012. 
33. Patrick AN, Cabrera JH, Smith AL, Chen XS, Ford HL and Zhao R: Structure-function analyses of the human SIX1-EYA2 complex reveal insights into metastasis and BOR syndrome. Nat Struct Mol Biol 20: 447-453, 2013.

34. Ohto H, Kamada S, Tago K, Tominaga SI, Ozaki H, Sato S and Kawakami K: Cooperation of six and eya in activation of their target genes through nuclear translocation of Eya. Mol Cell Biol 19: 6815-6824, 1999.

35. Grifone R, Demignon J, Giordani J, Niro C, Souil E, Bertin F, Laclef C, Xu PX and Maire P: Eya1 and Eya2 proteins are required for hypaxial somitic myogenesis in the mouse embryo. Dev Biol 302: 602-616, 2007.

36. Embry AC, Glick JL, Linder ME and Casey PJ: Reciprocal signaling between the transcriptional co-factor Eya2 and specific members of the Galphai family. Mol Pharmacol 66: 1325-1331, 2004.

37. Ali-Khan SE and Hales BF: Novel retinoid targets in the mouse limb during organogenesis. Toxicol Sci 94: 139-152, 2006.

38. Molinari M: Cell cycle checkpoints and their inactivation in human cancer. Cell Prolif 33: 261-274, 2000.

39. Fu J, Xu X, Kang L, Zhou L, Wang S, Lu J, Cheng L, Fan Z, Yuan B, Tian P, et al: miR-30a suppresses breast cancer cell proliferation and migration by targeting Eya2. Biochem Biophys Res Commun 445: 314-319, 2014.

40. Clark SW, Fee BE and Cleveland JL: Misexpression of the eyes absent family triggers the apoptotic program. J Biol Chem 277: 3560-3567, 2002.
41. Matt N, Dupé V, Garnier JM, Dennefeld C, Chambon P, Mark M and Ghyselinck NB: Retinoic acid-dependent eye morphogenesis is orchestrated by neural crest cells. Development 132: 4789-4800, 2005.

42. Li X, Oghi KA, Zhang J, Krones A, Bush KT, Glass CK, Nigam SK, Aggarwal AK, Maas R, Rose DW, et al: Eya protein phosphatase activity regulates Six1-Dach-Eya transcriptional effects in mammalian organogenesis. Nature 426: 247-254, 2003.

43. Kohrt D, Crary J, Zimmer M, Patrick AN, Ford HL, Hinds PW and Grossel MJ: CDK6 binds and promotes the degradation of the EYA2 protein. Cell Cycle 13: 62-71, 2014.

44. Bonini NM, Leiserson WM and Benzer S: The eyes absent gene: Genetic control of cell survival and differentiation in the developing Drosophila eye. Cell 72: 379-395, 1993.

45. Pandey RN, Rani R, Yeo EJ, Spencer M, Hu S, Lang RA and Hegde RS: The Eyes Absent phosphatase-transactivator proteins promote proliferation, transformation, migration, and invasion of tumor cells. Oncogene 29: 3715-3722, 2010.

46. Krueger AB, Drasin DJ, Lea WA, Patrick AN, Patnaik S, Backos DS, Matheson CJ, Hu X, Barnaeva E, Holliday MJ, et al: Allosteric inhibitors of the Eya2 phosphatase are selective and inhibit Eya2-mediated cell migration. J Biol Chem 289: 16349-16361, 2014. 